\title{
Radiative type-I seesaw neutrino masses
}

\author{
Carolina Arbeláez, ${ }^{1, *}$ A. E. Cárcamo Hernández, ${ }^{1, \dagger}$ Ricardo Cepedello®, ${ }^{2, \ddagger}$ Martin Hirsch, ${ }^{2, \S}$ and Sergey Kovalenko ${ }^{1, \|}$ \\ ${ }^{1}$ Department of Physics, Universidad Técnica Federico Santa María, \\ Avenida España 1680 Valparaíso, Chile \\ ${ }^{2}$ AHEP Group, Instituto de Física Corpuscular-CSIC/Universitat de València Edificio de Institutos de \\ Paterna, Apartado 22085, E-46071 València, Spain
}

(Received 15 October 2019; published 9 December 2019)

\begin{abstract}
We discuss a radiative type-I seesaw. In these models, the radiative generation of Dirac neutrino masses allows to explain the smallness of the observed neutrino mass scale for rather light right-handed neutrino masses in a type-I seesaw. We first present the general idea in a model-independent way. This allows us to estimate the typical scale of right-handed neutrino mass as a function of the number of loops. We then present two example models, at the one- and two-loop level, which we use to discuss neutrino masses and lepton-flavor-violating constraints in more detail. For the two-loop example, right-handed neutrino masses must lie below $100 \mathrm{GeV}$, thus making this class of models testable in heavy neutral lepton searches.
\end{abstract}

DOI: 10.1103/PhysRevD.100.115021

\section{INTRODUCTION}

The simplest possibility to generate the Weinberg operator [1],

$$
\mathcal{O}^{W}=\frac{1}{\Lambda} L L H H
$$

is certainly the type-I seesaw mechanism [2-4] given by the diagram in Fig. 1. In the classical type-I seesaw the Yukawa vertices are point-like $\left(Y_{\nu} \bar{L} H \nu_{R}\right)$ and the smallness of the neutrino masses is controlled by the large Majorana mass $\left(\Lambda \sim M_{R}\right)$ of the right-handed neutrinos $\nu_{R}$.

After electroweak symmetry breaking with the Higgs vacuum expectation value (VEV), $v \equiv\left\langle H^{0}\right\rangle$, the Weinberg operator (1) leads to the light active neutrino Majorana mass terms. In one-generation notation, the active neutrino mass is then given by the well-known relation

$$
m_{\nu} \approx m_{D}^{2} / M_{R}, \quad \text { with } \quad m_{D}=Y_{\nu}\left\langle H^{0}\right\rangle .
$$

Assuming that the Yukawas entering $m_{D}$ take values of order $\mathcal{O}(1)$, current neutrino data [5] would then point to

\footnotetext{
*carolina.arbelaez@usm.cl

†antonio.carcamo@usm.cl

*icepe@ific.uv.es

\$mahirsch@ific.uv.es

"sergey.kovalenko@usm.cl
}

Published by the American Physical Society under the terms of the Creative Commons Attribution 4.0 International license. Further distribution of this work must maintain attribution to the author(s) and the published article's title, journal citation, and DOI. Funded by SCOAP ${ }^{3}$.
$M_{R} \sim 10^{(14-15)} \mathrm{GeV}$. This setup, apart from being able to explain neutrino oscillation data, leads to only one experimentally "testable" prediction: neutrinoless double-beta decay should be observed at some level (for reviews on $0 \nu \beta \beta$ decay see, for example, Refs. [6,7]).

Here, we instead discuss a simple idea that allows for a much lower scale $M_{R}$, even for all involved Yukawa couplings of order $\mathcal{O}(1)$, by effectively generating the Dirac mass term corresponding to the Yukawa vertices in Fig. 1. To this end, one can claim that the elementary Yukawa coupling is forbidden by some symmetry, which being softly broken allows one to generate these vertices at a certain loop level directly or via higher-dimensional effective operators of the form

$$
\frac{\kappa}{M^{2 n}} \bar{L} H \nu_{R}\left(H^{\dagger} H\right)^{n}
$$

where $M$ is the scale of new physics underlying these operators (supposedly somewhere above the electroweak scale) and $\kappa$ is a loop-suppression factor. The Dirac mass term is generated by the operator (3) after the electroweak symmetry breaking. We assume that only the standard model (SM) Higgs acquires a VEV, though it is straightforward to

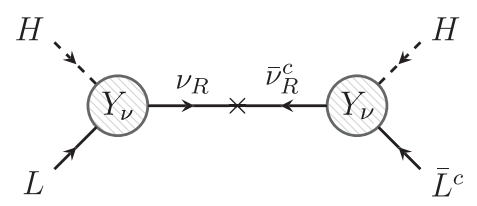

FIG. 1. Effective type-I seesaw. The neutrino mass is suppressed by the Majorana mass of $\nu_{R}$ and by the square of the Dirac Yukawa term $Y_{\nu}$ which is generated effectively; see Eq. (4). 
generalize this to non-SM Higgses with VEVs as well. Then, the resulting effective Yukawa couplings would be suppressed as

$$
Y_{\nu} \sim\left(\frac{1}{16 \pi^{2}}\right)^{\ell}\left(\frac{v^{2}}{M^{2}}\right)^{n}
$$

where $\ell$ is the number of loops in the diagram generating the operator (3).

As $Y_{\nu}$ is generated effectively, it can be naturally small, while all couplings arising in the UV-complete theory can take values of order $\mathcal{O}(1)$.

As shown in the next section, right-handed neutrino masses of order the electroweak scale are quite possible in this setup. Such moderately heavy right-handed neutrinos could be searched for in accelerator-based experiments via displaced vertices. The topic of "long-lived light particles" has attracted much attention in the recent literature [8]. A number of recent experimental proposals [9-12] could search for this signal. Sensitivity estimates for righthanded neutrinos for these experiments can be found in Refs. [13,14], and those for the main LHC experiments can be found in Refs. [15-17].

As we already mentioned, in order to forbid tree-level Dirac Yukawa couplings, it is necessary to postulate some additional symmetry beyond the SM gauge group. This symmetry could be either gauged or discrete. For simplicity, in our model constructions we will concentrate on discrete symmetries, starting with a $Z_{4}$ symmetry that gets softly broken to an exact remnant $Z_{2}$ symmetry. Thus, the same symmetry responsible for explaining the smallness of the neutrino mass is able to stabilize a dark matter candidate too.

In our setup neutrinos are Majorana particles. However, our constructions have some overlap with papers on Dirac neutrinos. The possibility that Dirac neutrino masses are small because they are radiatively generated has been considered already in the pioneering works of Refs. [18-22]. Some general considerations on how to obtain small Dirac neutrino masses have been discussed in Ref. [23]. Systematic studies of one-loop and two-loop Dirac neutrino masses were given in Refs. [24] and [25], respectively. Also the generation of $d=6$ Dirac neutrino masses has been considered [26].

Closer to our work are the neutrino mass models presented in Refs. [27,28]. As in our current paper, in these models neutrinos are Majorana particles with radiatively generated Dirac mass terms. However, both of these papers presented just one particular one-loop example model, while we formulate general conditions for the implementation of the radiative seesaw type-I mechanism at any loop order. We also describe in more detail two specific models at the one- and two-loop level. Moreover, different from the example model in Refs. [27,28], our models also have a candidate for cold dark matter.
The rest of this paper is organized as follows. In the next section we discuss the radiative generation of neutrino Dirac couplings from a model-independent point of view. This allows us to estimate the typical scales for the Majorana mass of neutrinos as a function of the loop level, at which the Dirac couplings are generated. In Sec. III we discuss two concrete example models at the one-loop and two-loop level. We estimate the neutrino masses for these models in more detail, discuss possible constraints from lepton flavor violation, and then turn briefly to dark matter. We then close with a short summary and outlook.

\section{RADIATIVE TYPE-I SEESAW}

In this brief section we discuss the radiative generation of neutrino Dirac Yukawa couplings from a modelindependent point of view. Here we consider only the $d=4$ Dirac mass operator $L H \bar{\nu}_{R}$ generated via loops. The mass of the light active neutrinos arises from the diagram depicted in Fig. 1 and is given by Eq. (2).

For simplicity, we limit ourselves to discussing the phenomenologically unrealistic case of one massive neutrino with no hierarchy or flavor structure for the Yukawas. This is sufficient for discussing the parameter dependence, and extending to three generations of active neutrinos is straightforward. The Dirac Yukawa $Y_{\nu}$ can be parametrized in general in terms of five exponents $(\ell, \alpha, \beta, \gamma, \delta) \in \mathbb{N}^{+}$, whose values will depend on the specific UV-complete realization of the operator $Y_{\nu} L H \bar{\nu}_{R}$ as

$$
Y_{\nu} \sim\left(\frac{1}{16 \pi^{2}}\right)^{\ell}\left(\frac{m_{\tau}}{v}\right)^{\alpha}\left(\frac{M_{F}}{\Lambda}\right)^{\beta}\left(\frac{\mu}{\Lambda}\right)^{\gamma} \epsilon^{\delta} .
$$

This corresponds to effectively generating the Yukawa via a diagram with the following features:

(1) $\ell$ loops.

(2) $\alpha$ insertions of SM Yukawas. Unless the UV model allows for a top quark in the loop, this corresponds to a suppression of typically $\sim 10^{-2}$ from $Y_{\tau}^{\mathrm{SM}}$ (or $Y_{b}^{\mathrm{SM}}$ ).

(3) $\beta$ mass insertions of new (vector-like) fermions that are not part of the SM, all set to $M_{F}$ for simplicity.

(4) $\gamma$ dimensionful couplings in the scalar sector, i.e., trilinear scalar couplings.

(5) $\delta$ dimensionless couplings, such as Yukawas or fourpoint scalar couplings.

For a UV-complete model which is genuine, i.e., gives the dominant contribution to the neutrino mass, the possible sets of values of these exponents are limited. For example, for the most simple case of a one-loop Dirac mass term, there are only two genuine diagrams [24] with one or two mass insertions and, at least, three couplings. So, for $\ell=1$ it is not possible to generate a genuine diagram with, for instance, $\alpha, \beta>2$. The possible combinations of $(\alpha, \beta, \gamma, \delta)$ can be deduced from the systematic studies of radiative Dirac models given in Refs. [24,25]. 
For our numerical estimates, we assume that all couplings are in the perturbative regime, i.e., $\epsilon \lesssim 1$. $^{1}$ If $\mu$ is a trilinear coupling between some beyond-the-SM scalar and the Higgs, it enters in the calculation of the stability of the Higgs potential, i.e., it will induce a modification of the quartic Higgs coupling at the one-loop level. Thus, we also assume that $\mu \lesssim m_{S} \equiv \epsilon m_{S}$ in order to not run into problems with the SM Higgs sector. With these considerations the light neutrino mass can be written in terms of the same five exponents, using the seesaw relation (2),

$m_{\nu} \sim\left(\frac{1}{16 \pi^{2}}\right)^{2 \ell} \frac{v^{2}}{M_{R}}\left(\frac{m_{\tau}}{v}\right)^{2 \alpha}\left(\frac{M_{F}}{\Lambda}\right)^{2 \beta}\left(\frac{m_{S}}{\Lambda}\right)^{2 \gamma} \epsilon^{2(\gamma+\delta)}$.

As this equation shows, neutrino masses generated from this class of models will be very suppressed. If, for instance, the Dirac neutrino mass arises at two-loop order, then $m_{\nu}$ will effectively come from a four-loop diagram with an extra suppression due to the Majorana scale $M_{R}$. Thus, for relatively low masses of the order of $\mathrm{TeV}$ and couplings of order one, a reasonable neutrino mass can be easily obtained.

A rough but conservative limit on the Majorana mass scale can be obtained setting all masses in the loop to the same scale $\Lambda=M_{F}=m_{S}$. Conservatively taking $\epsilon=1$, we find

$$
m_{\nu} \sim\left(\frac{1}{16 \pi^{2}}\right)^{2 \ell} \frac{v^{2}}{M_{R}}\left(\frac{m_{\tau}}{v}\right)^{2 \alpha} .
$$

Note that the scale $\Lambda$ does not appear in this simple case in the expression for $m_{\nu}$. This is to be expected, given the $d=4$ nature of the neutrino Dirac coupling. Taking as a reference scale the atmospheric neutrino mass $\sqrt{\left|\Delta m_{31}^{2}\right|} \approx 0.05 \mathrm{eV}$, we can set upper limits on $M_{R}$ as a function of the exponents $\ell$ and $\alpha$. The limits are given in Table I up to three loops and two SM Yukawa insertions. The numbers given correspond to couplings of order one.

Obviously, $M_{R}$ decreases very fast as $\alpha$ or $\ell$ increases. This is due to the fact that for Majorana neutrinos $m_{\nu}$ depends quadratically on $Y_{\nu}$, rather than linearly. For $\alpha=1$ and $l=2$ one finds a scale of $M_{R} \sim 10^{2} \mathrm{GeV}$, and similar values for $\alpha=2$ and $l=1$ or $\alpha=0$ and $l=3$. These are phenomenologically the most interesting cases.

Apart from the upper limit on the Majorana mass coming from the neutrino mass scale, lower limits on $M_{R}$ can be set from big bang nucleosynthesis [29] and the effective number of neutrinos in the early Universe $\Delta N_{\text {eff }}$ [30]. These limits depend on the mixing angle between the right-handed and active neutrinos (as a function of the

\footnotetext{
${ }^{1}$ It is often argued that perturbativity only requires Yukawa couplings to be $Y \lesssim \sqrt{4 \pi}$. However, saturating this limit would imply that higher-order contributions are (at least) equally important than the leading order (that we consider), thus rendering estimates effectively inconsistent.
}

TABLE I. Estimated values for $M_{R}$ needed to fit a neutrino mass of $0.05 \mathrm{eV}$ with couplings of order one for different realizations of the Dirac mass operator $L H \bar{\nu}_{R}$, considering $\ell$ loops and $\alpha$ SM Yukawa insertions. These mass scales constitute a rough but conservative upper limit for $M_{R}$ for each class of models parametrized by the exponents $\ell$ and $\alpha$ in Eq. (7).

\begin{tabular}{lccc}
\hline \hline$M_{R}$ & $\alpha=0$ & $\alpha=1$ & $\alpha=2$ \\
\hline$\ell=1$ & $2 \times 10^{10} \mathrm{GeV}$ & $2 \times 10^{6} \mathrm{GeV}$ & $2 \times 10^{2} \mathrm{GeV}$ \\
$\ell=2$ & $10^{6} \mathrm{GeV}$ & $10^{2} \mathrm{GeV}$ & $9 \times 10^{-3} \mathrm{GeV}$ \\
$\ell=3$ & $4 \times 10^{1} \mathrm{GeV}$ & $4 \times 10^{-3} \mathrm{GeV}$ & $4 \times 10^{-7} \mathrm{GeV}$ \\
\hline \hline
\end{tabular}

mass $\left.M_{R}\right)$. For our class of models, as for the ordinary type-I seesaw, one expects $M_{R} \gtrsim(0.1-1) \mathrm{GeV}$ from these considerations $[29,30]$. This significantly constrains the space of possible models to only those with three loops or less and at most two SM mass insertions (for the one-loop case). Therefore, in the next section we will discuss two model examples in more detail: a one-loop model and a two-loop model.

\section{EXAMPLES OF MODELS}

In this section we show two simple models where the Dirac mass is generated at one and two loops, both containing a stable dark matter candidate that participates in the loop. We give an estimate of the neutrino mass scale involved for a simplified benchmark, as well as insight into the phenomenological constraints coming from charged lepton-flavor-violating processes.

\section{A. One-loop Dirac mass}

The particle spectrum of the model and their assignments under the SM gauge and $Z_{4}$ discrete symmetry are shown in Table II. Notice that we have assumed a $Z_{4}$ symmetry, which is softly broken down to the preserved $Z_{2}$ symmetry, in order to guarantee that the Dirac neutrino mass matrix is generated at the one-loop level. The scalar sector of the model is composed of the SM Higgs doublet $H$, the inert $S U(2)_{L}$ scalar doublet $\eta$, and the electrically charged gauge singlet scalar $S^{-}$. In addition, the SM fermion sector is extended by the inclusion of a right-handed Majorana neutrino $\nu_{R}{ }^{2}$ and the vector-like charged leptons $\chi_{L}$ and $\chi_{R}$. The relevant terms for the neutrino mass take the forms

$$
\begin{gathered}
-\mathcal{L}_{Y}=Y_{e} L H^{\dagger} e^{c}+Y_{L} L \eta^{\dagger} \chi_{L}+Y_{R} \overline{\chi_{R}} S^{+} \overline{\nu_{R}}+\text { H.c. }, \\
\mathcal{L}_{M}=M_{R} \overline{\nu_{R}^{c}} \nu_{R}+M_{\chi} \overline{\chi_{R}} \chi_{L}+\text { H.c. },
\end{gathered}
$$

\footnotetext{
${ }^{2}$ We repeat that here we are only interested in a rough estimate for the neutrino mass scale. For phenomenological reasons, one would need at least two right-handed neutrinos that generate the solar and atmospheric neutrino mass. Since fits of the type-I seesaw to neutrino data are straightforward and have been done many times in the literature, we do not repeat these details here.
} 
TABLE II. Particle content of the example model that generates the one-loop diagram of Fig. 2 once the $Z_{4}$ is softly broken by the trilinear term $H \eta S^{-}$. After the breaking of $Z_{4}$ a remnant $Z_{2}$ is exactly conserved.

\begin{tabular}{lccc}
\hline \hline Fields & $S U(3)_{C} \times S U(2)_{L} \times U(1)_{Y}$ & $Z_{4}$ & Residual $Z_{2}$ \\
\hline$L$ & $(\mathbf{1}, \mathbf{2},-1 / 2)$ & 1 & 1 \\
$e^{c}$ & $(\mathbf{1}, \mathbf{1}, 1)$ & 1 & 1 \\
$\nu_{R}$ & $(\mathbf{1}, \mathbf{1}, 0)$ & -1 & 1 \\
$H$ & $(\mathbf{1}, \mathbf{2}, 1 / 2)$ & 1 & 1 \\
$\left(\chi_{L}, \chi_{R}\right)$ & $(\mathbf{1}, \mathbf{1}, 1)$ & $(i, i)$ & $(-1,-1)$ \\
$\eta$ & $(\mathbf{1}, \mathbf{2}, 1 / 2)$ & $i$ & -1 \\
$S^{-}$ & $(\mathbf{1}, \mathbf{1},-1)$ & $i$ & -1 \\
\hline \hline
\end{tabular}

where flavor indices and $S U(2)$ contractions have been suppressed for brevity.

The terms above generate the Dirac neutrino mass matrix at the one-loop level through the diagram shown in Fig. 2 provided the following $Z_{4}$ trilinear soft-breaking term is added to the scalar potential:

$$
\mathcal{V} \supset \mu_{S} H \eta S^{-}+\text {H.c. }
$$

The softly broken $Z_{4}$ guarantees that the Dirac mass term is forbidden at the tree level but generated by loops, i.e., that the diagram is genuine (irreducible) [24].

The Dirac mass term can be computed directly from the diagram in Fig. 2 given the Lagrangians (8) and (9) and the soft-breaking term. In the mass insertion approximation and, for simplicity, setting all of the masses of the internal scalars as well as the soft-breaking parameter $\mu_{S}$ to $m_{S}$, one finds

$$
m_{D} \approx \frac{1}{16 \pi^{2}} \frac{v m_{S}}{M_{\chi}} Y_{L} Y_{R} \mathcal{I}_{1}\left(m_{S}^{2} / M_{\chi}^{2}\right)
$$

The loop integral $\mathcal{I}_{1}(x)$ can be written in terms of the Passarino-Veltman $B_{0}$ function [31] as

$$
\mathcal{I}_{1}(x)=\frac{1}{1-x}\left[B_{0}(0,1, x)-B_{0}(0, x, x)\right]
$$

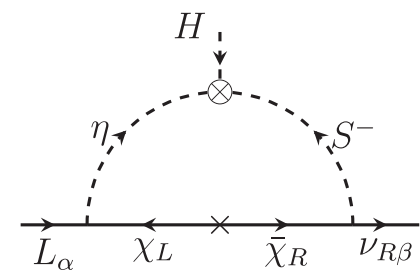

FIG. 2. One-loop Dirac neutrino mass. The diagram is realized when the $Z_{4}$ is softly broken (denoted by the symbol $\otimes$ ). As the symmetry is broken into two units, the diagram is still invariant under a remnant $Z_{2}$ of $Z_{4}$.
The mass scale of the lightest active neutrino can be directly estimated through the seesaw approximation as

$$
m_{\nu} \sim\left(\frac{1}{16 \pi^{2}}\right)^{2} Y_{L}^{2} Y_{R}^{2} \frac{v^{2} m_{S}^{2}}{M_{\chi}^{2} M_{R}}\left[\mathcal{I}_{1}\left(m_{S}^{2} / M_{\chi}^{2}\right)\right]^{2}
$$

This mass scale as a function of $M_{R}$ is plotted in Fig. 3. Two different benchmarks with $M_{\chi}=M_{R}$ and $m_{S}=M_{\chi}$ are represented by the solid and dashed lines, respectively. For both cases, we can observe that the neutrino mass is strongly suppressed even for small values of $M_{R}$. In the $m_{S}=M_{\chi}$ scenario, the neutrino mass falls as $\sim 1 / M_{R}$ independently of the one-loop internal scalar masses. Moreover, in the $M_{\chi}=M_{R}$ scenario the neutrino mass is a function of both mass scales $m_{S}$ and $M_{R}$. It behaves as $M_{R}$ or $1 / M_{R}^{3}$ depending on which of these two scales dominates the loop.

The window of allowed $M_{R}$ values that could fit the neutrino oscillation scale $m_{\nu} \sim 0.05 \mathrm{eV}$ becomes narrower for larger masses $m_{S}$. Note that in Fig. 3 the neutrino mass is plotted for order-one couplings. Consequently, the points with a neutrino mass lying roughly below the atmospheric scale are phenomenologically nonviable, as they would require couplings larger than one (nonperturbative) to give a reasonable mass scale.

Current upper limits on lepton-flavor-violating (LFV) decays such as $\mu \rightarrow e \gamma$ can provide constraints on the

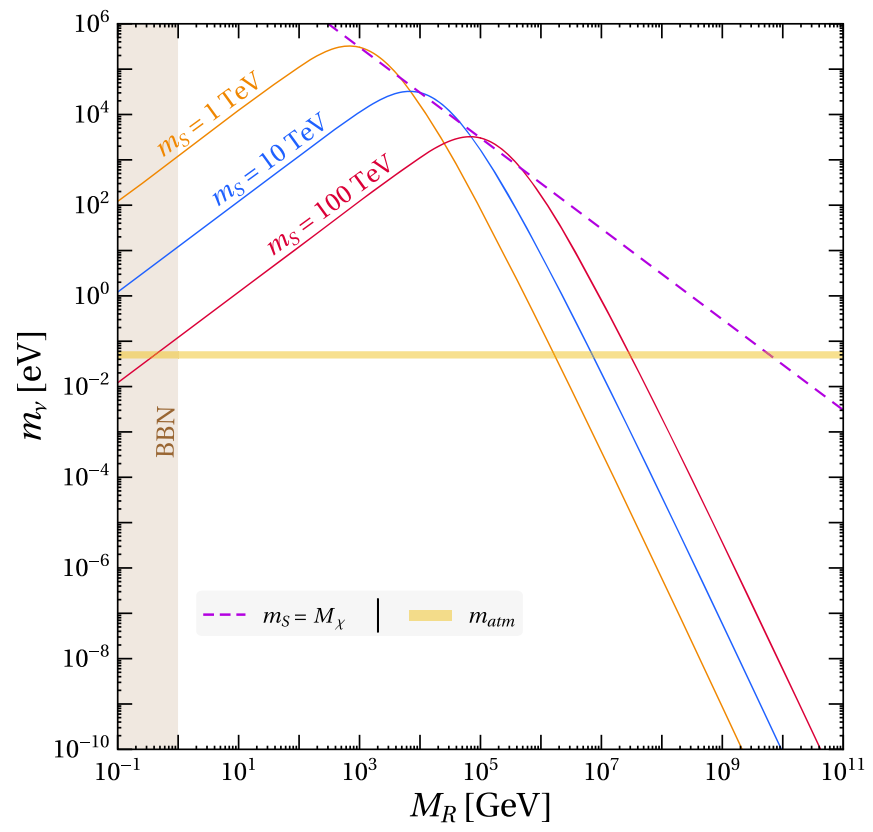

FIG. 3. One-loop neutrino mass scale. The dashed line corresponds to the case where $m_{S}=M_{\chi}$, while the solid lines depict the case where $M_{\chi}=M_{R}$ for different scalar masses. The Yukawas $Y_{L}$ and $Y_{R}$ are set to 1 . Big bang nucleosynthesis (BBN) excludes $M_{R}>(0.1-1) \mathrm{GeV}$, depending on mixing, for this class of models [29]. 
parameters of our model. These depend on specific choices for the Yukawas $Y_{L}$ and $Y_{R}$. As Eq. (13) shows, $m_{\nu}$ depends on the product of these couplings, while LFV decays are mostly sensitive to only $Y_{L}$. There are then two extreme cases: (i) choose $Y_{L} \simeq 1$ and fit $Y_{R}$ to $m_{\nu}$ as a function of the other model parameters, or (ii) choose $Y_{R} \simeq 1$ and fit $Y_{L}$. Case (i) is very similar to the situation in our two-loop model (see Sec. III B), and thus we will discuss the details in the next section. For case (ii), on the other hand, we found that LFV limits do not impose interesting limits on our one-loop model.

The residual $Z_{2}$ symmetry ensures that the lightest of the fields running inside the loop will be stable. In order to not conflict with cosmology and to provide a good dark matter candidate, one should force the neutral component of the doublet $\eta$ to be the lightest of the loop particles. Similar DM candidates have been studied in the literature. ${ }^{3}$ Considering $\eta$ as the only source of dark matter, the observed relic density, together with direct-detection limits and the constraints on the invisible width of the Higgs boson, severely limit its mass to lie either around $m_{h} / 2 \simeq 62.5 \mathrm{GeV}$, in a small region around $m_{\eta} \simeq 72 \mathrm{GeV}$, or above $m_{\eta} \gtrsim$ $500 \mathrm{GeV}$ [34].

\section{B. Two-loop Dirac mass}

Analogously to the first example, we build a two-loop radiative seesaw model that softly breaks a $Z_{4}$ discrete group to an exact $Z_{2}$ symmetry. The particle content and their transformation properties under the SM gauge and the $Z_{4}$ discrete symmetry are shown in Table III. We again include a right-handed Majorana neutrino $\nu_{R}$.

The relevant terms of the Lagrangian and the scalar sector invariant under $Z_{4}$ are

$$
\begin{gathered}
-\mathcal{L}_{Y}=Y_{e} L H^{\dagger} e^{c}+Y_{L} \overline{F_{L}} \eta_{2} \overline{e^{c}}+Y_{R} \overline{\nu_{R}} \eta_{2} F_{L}+\text { H.c. } \\
\mathcal{L}_{M}=M_{R} \overline{\nu_{R}^{c}} \nu_{R}+M_{F} \overline{F_{R}} F_{L}+\text { H.c. } \\
\mathcal{V} \supset \lambda \eta_{1}^{\dagger} H \eta_{2}^{\dagger} H+\text { H.c. }
\end{gathered}
$$

An effective Dirac term is generated once the $Z_{4}$ symmetry is softly broken in the scalar sector by the term

$$
-\mathcal{L}_{\text {soft }}=\mu_{12}^{2} \eta_{2}^{\dagger} \eta_{1}+\text { H.c. }
$$

A Dirac mass appears at the two-loop level, as depicted in Fig. 4, which can be expressed in the mass insertion approximation, assuming no flavor structure in the Yukawa couplings, as

\footnotetext{
${ }^{3}$ See, for instance, the well-known inert doublet model [32] or the scotogenic model [33].
}

TABLE III. Particle content of the example model that generates the two-loop diagram of Fig. 4 once the $Z_{4}$ is softly broken by the term $\eta_{2}^{\dagger} \eta_{1}$. After the breaking of $Z_{4}$ a remnant $Z_{2}$ is conserved.

\begin{tabular}{lccc}
\hline \hline Fields & $S U(3)_{C} \times S U(2)_{L} \times U(1)_{Y}$ & $Z_{4}$ & Residual $Z_{2}$ \\
\hline$L$ & $(\mathbf{1}, \mathbf{2},-1 / 2)$ & 1 & 1 \\
$e^{c}$ & $(\mathbf{1}, \mathbf{1}, 1)$ & 1 & 1 \\
$\nu_{R}$ & $(\mathbf{1}, \mathbf{1}, 0)$ & -1 & 1 \\
$H$ & $(\mathbf{1}, \mathbf{2}, 1 / 2)$ & 1 & 1 \\
$\left(F_{L}, F_{R}\right)$ & $(\mathbf{1}, \mathbf{2},-1 / 2)$ & $(i, i)$ & $(-1,-1)$ \\
$\eta_{1}$ & $(\mathbf{1}, \mathbf{2}, 1 / 2)$ & $-i$ & -1 \\
$\eta_{2}$ & $(\mathbf{1}, \mathbf{2}, 1 / 2)$ & $i$ & -1 \\
\hline \hline
\end{tabular}

$$
m_{D} \approx\left(\frac{1}{16 \pi^{2}}\right)^{2} \lambda Y_{e} Y_{L} Y_{R} \frac{v \mu_{12}^{2}}{M_{F}^{2}} \mathcal{I}_{2}\left(m_{S}^{2} / M_{F}^{2}\right)
$$

where $\mathcal{I}_{2}(x)$ is a dimensionless two-loop function. $\mu_{12}$ is the soft-breaking mass term depicted by $\otimes$ in Fig. 4. For simplicity, we set all of the masses of the new internal scalars to $m_{S}$. Taking into account that the main contribution of the SM Yukawa $Y_{e}$ would be $m_{\tau} / v$, the mass scale of the lightest active neutrino is directly estimated through the seesaw approximation as

$$
m_{\nu} \sim\left(\frac{1}{16 \pi^{2}}\right)^{4} \lambda^{2} Y_{L}^{2} Y_{R}^{2} \frac{m_{\tau}^{2} m_{S}^{4}}{M_{F}^{4} M_{R}}\left[\mathcal{I}_{2}\left(m_{S}^{2} / M_{F}^{2}\right)\right]^{2},
$$

where, as before, we have set $\mu_{12}=m_{S}$. $\mathcal{I}_{2}$ can be written in terms of simple two-loop integrals for which analytical solutions are known [35]. We do not give its decomposition here for brevity, though it can be found in the literature [36].

The neutrino mass scale (19) as a function of $M_{R}$ is plotted in Fig. 5. We consider two different approximations, $M_{F}=m_{S}$ and $M_{F}=M_{R}$, represented by the dashed and solid lines, respectively. As expected from Table I, the neutrino mass is more strongly suppressed compared to the one-loop model described previously. For the case $m_{S}=$ $M_{F}$ the Dirac Yukawa is independent of the scale, and

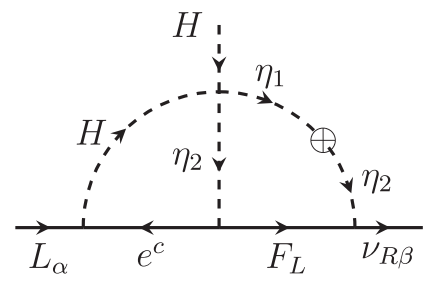

FIG. 4. Two-loop Dirac neutrino mass. The diagram is realized when the $Z_{4}$ is softly broken (denoted by the symbol $\otimes$ ). As the symmetry is broken into two units, the diagram is still invariant under a remnant $Z_{2}$. 


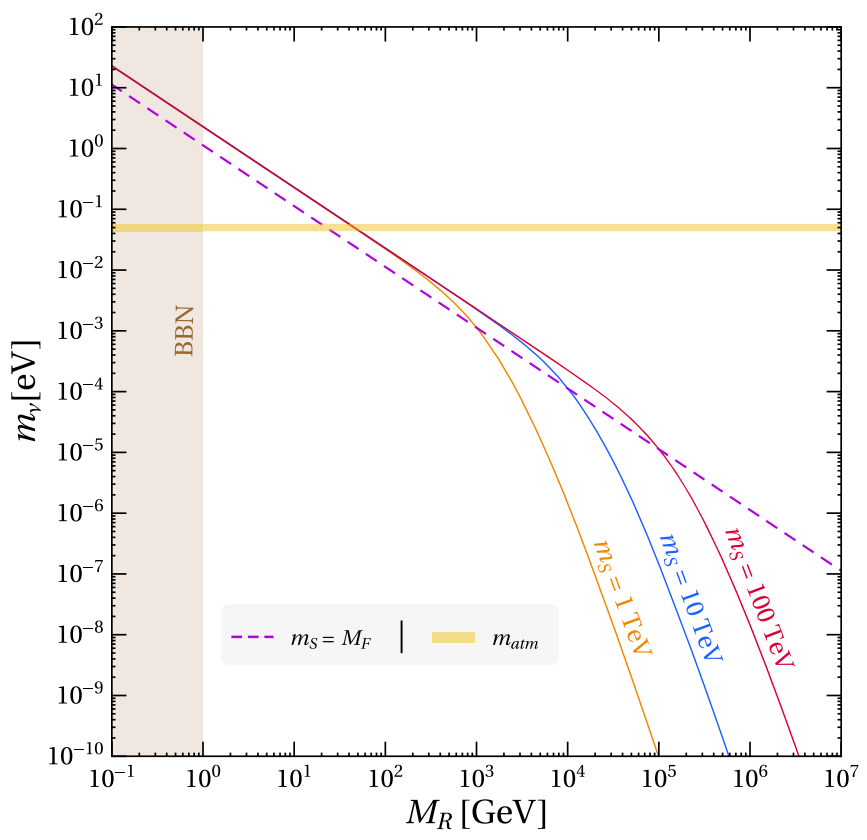

FIG. 5. Two-loop neutrino mass scale assuming that $m_{S}=M_{F}$ and $M_{F}=M_{R}$, depicted as dashed and solid lines, respectively. All dimensionless couplings are set to 1 and the BBN exclusion region is indicated on the left.

consequently the neutrino mass falls simply as $\sim 1 / M_{R}$. On the other hand, in the scenario where $M_{F}=M_{R}$ this same behavior is reproduced when $m_{S}$ dominates, while for values of $M_{R}>m_{S}$ the neutrino mass follows the curve $1 / M_{R}^{5}$.

Given the suppression factor $\left(m_{\tau} / v\right)^{2} \sim 10^{-4}$, and if we take into account the limit coming from cosmology (BBN), the range of allowed values of $M_{R}$ that can fit the neutrino oscillation scale $m_{\mathrm{atm}} \sim 0.05 \mathrm{eV}$ is considerably limited. For $m_{S}>10^{2} \mathrm{GeV}, M_{R}$ has to be $M_{R} \lesssim 10^{2} \mathrm{GeV}$. This makes the model testable in future heavy neutral lepton searches.

We mention again that the remnant $Z_{2}$ symmetry stabilizes the lightest of the fields that are odd under this symmetry. Fermionic dark matter coming from a doublet is ruled out by direct-detection experiments [37], while for the scalar inert doublet the same limits described in the previous section apply.

Turning to LFV processes, Fig. 6 shows $\operatorname{Br}(\mu \rightarrow e \gamma)$ as a function of $M_{R}$ for two different scenarios already mentioned in Sec. III A: (i) choose $Y_{L} \simeq 1$ and fit $Y_{R}$ to $m_{\nu}$, or (ii) choose $Y_{R} \simeq 1$ and fit $Y_{L}$. All other possibilities Yukawas choices lie between these extremes. The dominant (one-loop) contribution to $\operatorname{Br}(\mu \rightarrow e \gamma)$ always comes from $Y_{L}$, which directly connects the new particles with the SM leptons. For $M_{F}=M_{R}$ and $Y_{R}=1$ the branching is dominated by the fit of the neutrino mass (19). The branching increases as a function of $M_{R}$ as $Y_{L}$ gets larger, counteracting the suppression of $1 / M_{R}^{5}$ in the neutrino

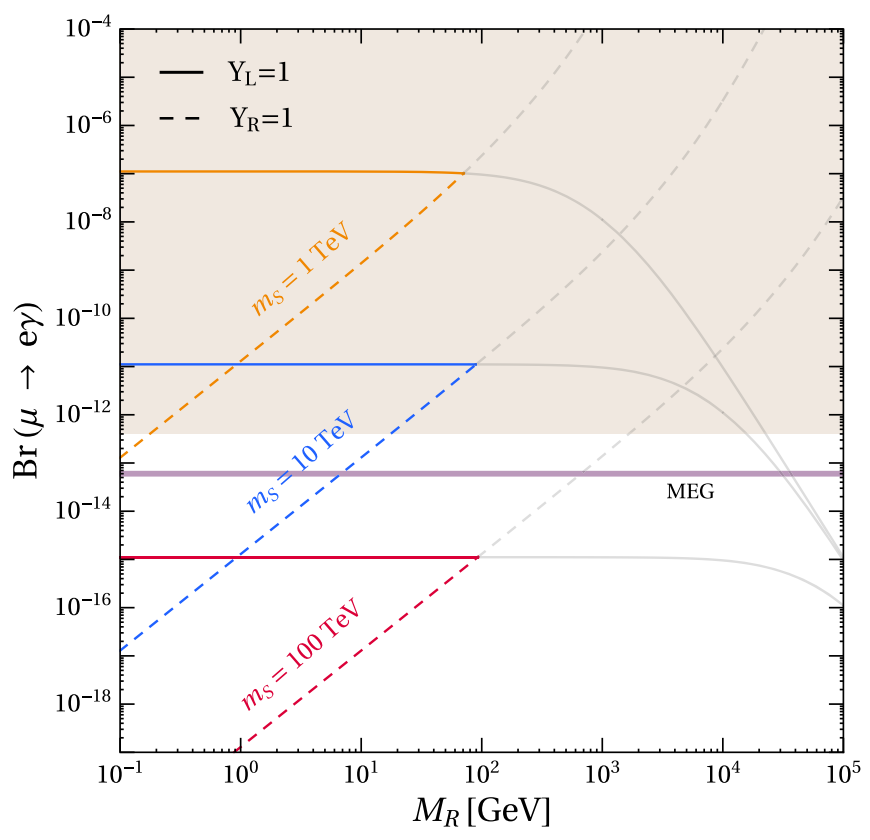

FIG. 6. Estimate of the branching ratio of $\mu \rightarrow e \gamma$ as a function of $M_{R}$ for different values of $m_{S}$ fitting the neutrino mass to $m_{\text {atm }}$. The areas between the colored lines are allowed in this model (see text). The grey lines represent the values of $M_{R}$ where one of the Yukawa couplings becomes nonperturbative in order to fit neutrino oscillation data. The shaded region represents the experimentally excluded area for $\operatorname{Br}(\mu \rightarrow e \gamma)>4.2 \times 10^{-13}$ [39], while the purple line corresponds to the future limit proposed by the MEG Collaboration [40].

mass. We stop the calculation when $Y_{L}$ grows larger than 1 . In contrast, for $Y_{L}=1$ there is no dependence from the neutrino mass fit, but rather a suppression of $1 / M_{R}^{4}$ when this mass scale dominates over $m_{S}$ in the $\mu \rightarrow e \gamma$ loop function [38]. The regions in between these extremes are the allowed regions for this neutrino mass model.

\section{CONCLUSIONS}

We have constructed a new realization of the type-I seesaw mechanism based on radiatively generated Dirac neutrino masses. We showed that this class of models can naturally generate a small neutrino mass for order-one couplings and relatively low mass scales. Compared to the standard type-I seesaw mechanism, for which the Majorana mass scale should be of the order of the grand unified theory scale, we found viable models even for $M_{R}$ below $100 \mathrm{GeV}$. Parametrizing the neutrino mass in terms of five integers, we derived a conservative limit on $M_{R}$ for each set of models, requiring only that they should fit the atmospheric neutrino mass scale. The strong suppression of the light neutrino mass with the number of loops, i.e., $\left(1 / 16 \pi^{2}\right)^{2 \ell}$, along with the seesaw Majorana mass suppression allows remarkably low $M_{R}$ values. This fact makes 
models with a large number of loops (or SM mass insertions) conflict with big bang nucleosynthesis and $\Delta N_{\text {eff }}$, which therefore significantly constrains the space of possible models.

To illustrate this idea in further detail, we presented two example models where the Dirac neutrino mass matrix is generated at the one- and two-loop level. The latter lies at the edge of the excluded models. An extra $Z_{4}$ symmetry is incorporated to forbid a tree-level Dirac mass, but it is broken softly in order to radiatively generate the Dirac Yukawa. A remnant exact $Z_{2}$ symmetry is kept that stabilizes the lightest of the $Z_{2}$ charged fields and provides a good dark matter candidate.

\section{ACKNOWLEDGMENTS}

C. A., A.E. C., and S. K. are supported by Fondecyt (Chile) Grants No. 11180722, No. 1170803, and No. 1190845, and CONICYT PIA/BASAL FB0821. R. C. and M. H. acknowledge funding by the Spanish grants Red Consolider MultiDark FPA2017-90566-REDC (MICINN), FPA2017-85216-P (MICINN), SEV-2014-23510398 (AEI/ FEDER, UE), PROMETEO/2018/165 (Generalitat Valenciana), and FPU15/03158. R. C. is also supported by Beca Santander Iberoamérica 2018/19 and would like to thank the Universidad Técnica Federico Santa María (USM) Department of Physics and Centro Científico Tecnológico de Valparaíso (CCTVal) for their hospitality.
[1] S. Weinberg, Phys. Rev. Lett. 43, 1566 (1979).

[2] P. Minkowski, Phys. Lett. 67B, 421 (1977).

[3] T. Yanagida, Workshop on the Baryon Number of the Universe and Unified Theories, Tsukuba, Japan, edited by O. Sawada and A. Sugamoto (National Lab, 1979), p. 109.

[4] R. N. Mohapatra and G. Senjanovic, Phys. Rev. Lett. 44, 912 (1980).

[5] P. F. de Salas, D. V. Forero, C. A. Ternes, M. Tortola, and J. W. F. Valle, Phys. Lett. B 782, 633 (2018).

[6] I. Avignone, T. Frank, S. R. Elliott, and J. Engel, Rev. Mod. Phys. 80, 481 (2008).

[7] F. F. Deppisch, M. Hirsch, and H. Pas, J. Phys. G 39, 124007 (2012).

[8] J. Alimena et al., arXiv:1903.04497.

[9] D. Curtin et al., Rep. Prog. Phys. 82, 116201 (2019).

[10] V. V. Gligorov, S. Knapen, M. Papucci, and D. J. Robinson, Phys. Rev. D 97, 015023 (2018).

[11] J. L. Feng, I. Galon, F. Kling, and S. Trojanowski, Phys. Rev. D 97, 035001 (2018).

[12] V. V. Gligorov, S. Knapen, B. Nachman, M. Papucci, and D. J. Robinson, Phys. Rev. D 99, 015023 (2019).

[13] J. C. Helo, M. Hirsch, and Z. S. Wang, J. High Energy Phys. 07 (2018) 056.

[14] D. Dercks, H. K. Dreiner, M. Hirsch, and Z. S. Wang, Phys. Rev. D 99, 055020 (2019).

[15] G. Cottin, J. C. Helo, and M. Hirsch, Phys. Rev. D 97, 055025 (2018).

[16] J. Liu, Z. Liu, L.-T. Wang, and X.-P. Wang, J. High Energy Phys. 07 (2019) 159.

[17] M. Drewes and J. Hajer, arXiv:1903.06100.

[18] T. P. Cheng and L.-F. Li, Phys. Rev. D 17, 2375 (1978).

[19] D. Chang and R. N. Mohapatra, Phys. Rev. Lett. 58, 1600 (1987).

[20] R. N. Mohapatra, Phys. Lett. B 201, 517 (1988).
[21] G. C. Branco and C. Q. Geng, Phys. Rev. Lett. 58, 969 (1987).

[22] P.-H. Gu and U. Sarkar, Phys. Rev. D 77, 105031 (2008).

[23] E. Ma and O. Popov, Phys. Lett. B 764, 142 (2017).

[24] C.-Y. Yao and G.-J. Ding, Phys. Rev. D 96, 095004 (2017); 98, 039901(E) (2018).

[25] S. Centelles Chuliá, R. Cepedello, E. Peinado, and R. Srivastava, J. High Energy Phys. 10 (2019) 093.

[26] S. Centelles Chuliá, R. Srivastava, and J. W. F. Valle, Phys. Rev. D 98, 035009 (2018).

[27] S. Nasri and S. Moussa, Mod. Phys. Lett. A 17, 771 (2002).

[28] S. Kanemura, T. Nabeshima, and H. Sugiyama, Phys. Lett. B 703, 66 (2011).

[29] F. F. Deppisch, P. S. Bhupal Dev, and A. Pilaftsis, New J. Phys. 17, 075019 (2015).

[30] S. Gariazzo, P. F. de Salas, and S. Pastor, J. Cosmol. Astropart. Phys. 07 (2019) 014.

[31] G. Passarino and M. J. G. Veltman, Nucl. Phys. B160, 151 (1979).

[32] N. G. Deshpande and E. Ma, Phys. Rev. D 18, 2574 (1978).

[33] E. Ma, Phys. Rev. Lett. 81, 1171 (1998).

[34] B. Eiteneuer, A. Goudelis, and J. Heisig, Eur. Phys. J. C 77, 624 (2017).

[35] S. P. Martin and D. G. Robertson, Phys. Rev. D 95, 016008 (2017).

[36] D. Aristizabal Sierra, A. Degee, L. Dorame, and M. Hirsch, J. High Energy Phys. 03 (2015) 040.

[37] M. Cirelli, N. Fornengo, and A. Strumia, Nucl. Phys. B753, 178 (2006).

[38] L. Lavoura, Eur. Phys. J. C 29, 191 (2003).

[39] A. M. Baldini et al. (MEG Collaboration), Eur. Phys. J. C 76, 434 (2016).

[40] A. M. Baldini et al., arXiv:1301.7225. 